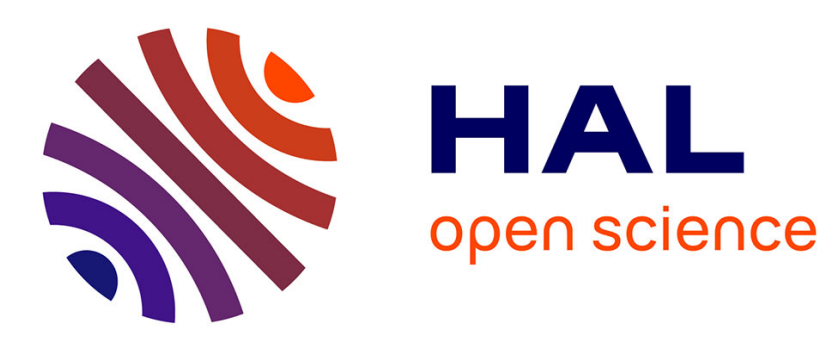

\title{
POLITIQUE CONTRACTUELLE ET STRATÉGIES UNIVERSITAIRES : LE RENDEZ-VOUS MANQUÉ?
}

Hervé Goy

\section{To cite this version:}

Hervé Goy. POLITIQUE CONTRACTUELlE ET STRATÉGIES UNIVERSITAIRES: LE RENDEZ-VOUS MANQUÉ? ?. Gestion et management public [2012-..], 2015, 3 (4), pp.65-82. halshs01215193

\section{HAL Id: halshs-01215193 \\ https://shs.hal.science/halshs-01215193}

Submitted on 13 Oct 2015

HAL is a multi-disciplinary open access archive for the deposit and dissemination of scientific research documents, whether they are published or not. The documents may come from teaching and research institutions in France or abroad, or from public or private research centers.
L'archive ouverte pluridisciplinaire HAL, est destinée au dépôt et à la diffusion de documents scientifiques de niveau recherche, publiés ou non, émanant des établissements d'enseignement et de recherche français ou étrangers, des laboratoires publics ou privés. 


\title{
POLITIQUE CONTRACTUELLE ET STRATÉGIES UNIVERSITAIRES : LE RENDEZ-VOUS MANQUÉ ?
} Hervé Goy

\author{
AIRMAP | «Gestion et management public »
}

2015/2 Volume $3 / \mathrm{n}^{\circ} 4$ | pages 65 à 82

Article disponible en ligne à l'adresse :

http://www.cairn.info/revue-gestion-et-management-public-2015-2-page-65.htm

!Pour citer cet article :

Hervé Goy, « Politique contractuelle et stratégies universitaires : le rendez-vous manqué ? », Gestion et management public 2015/2 (Volume 3 / n 4), p. 65-82.

Distribution électronique Cairn.info pour AIRMAP .

(C) AIRMAP. Tous droits réservés pour tous pays.

La reproduction ou représentation de cet article, notamment par photocopie, n'est autorisée que dans les limites des conditions générales d'utilisation du site ou, le cas échéant, des conditions générales de la licence souscrite par votre établissement. Toute autre reproduction ou représentation, en tout ou partie, sous quelque forme et de quelque manière que ce soit, est interdite sauf accord préalable et écrit de l'éditeur, en dehors des cas prévus par la législation en vigueur en France. Il est précisé que son stockage dans une base de données est également interdit. 


\title{
Politique contractuelle et stratégies universitaires : le rendez-vous manqué ${ }^{1}$
}

\author{
Hervé GOY²
}

\begin{abstract}
Résumé
Les universités contemporaines seraient devenues en une trentaine d'années des organisations entreprenantes, à même de développer des stratégies spécifiques. Concomitamment en France, la mise en œuvre d'une politique contractuelle ne semble pas avoir vraiment permis l'affirmation des stratégies universitaires. Cet article, fondé sur l'étude longitudinale du cas d'une université française, vise à en comprendre les raisons.
\end{abstract}

Mots clés : Stratégie, Université, Politique contractuelle, Isomorphisme institutionnel.

\section{Contractualization and universitary strategies: the missed opportunity?}

Abstract: Contemporary universities would become in thirty years entrepreneurial organizations, able to develop specific strategies. Simultaneously in France, the implementation of a contractual policy does not seem to really have confirmed the existence of such strategies within universities. This article, based on the longitudinal case study of a French university, aims to understand the reasons.

Key-words: Strategy, University, Contractual policy, Institutional isomorphism.

\section{Introduction}

" La contractualisation n'est pas encore identifiée comme un rendez-vous stratégique majeur par tous les établissements, alors qu'elle va de pair avec la formalisation de sa stratégie autour d'un projet fédérateur de la communauté universitaire ». Tel est l'un des constats dressé par un récent rapport du Sénat ${ }^{3}$, plus de vingt ans après la mise en œuvre de la politique contractuelle en France entre chaque université et son ministère de tutelle. Un tel bilan ne peut que nous interpeller, plusieurs années après la mise en œuvre d'importantes réformes des systèmes d'enseignement supérieur à l'échelle de I'Europe continentale (Bonaccorsi et Daraio, 2007), et alors même que les universités sont devenues l'un des vecteurs essentiels de l'économie de la connaissance (Delanty, 2001). Pour certains spécialistes des organisations universitaires pourtant (Clark, 1998 ; Etzkowitz et al., 2000 ; Askling,Kristensen, 2000 ; Davies, 2001), l'évolution rapide des caractéristiques des systèmes d'enseignement supérieur aurait dû obliger les universités à devenir davantage entreprenantes, les appelant en particulier à formuler les stratégies les mieux adaptées à leurs contextes et projets...

La question que nous proposons de traiter dans cet article est donc la suivante : pourquoi la contractualisation n'a-t-elle pas réussi à

\footnotetext{
Adresse électronique : herve.goy@univ-stetienne.fr

3 Page 62 du rapport d'information $n^{\circ} 446$ du 26 mars 2013 fait au nom de la commission sénatoriale pour le contrôle de l'application des lois sur la mise en œuvre de la loi n²007-1199 du 10 août 2007 relative aux libertés et responsabilités des universités, par Mme Dominique Gillot et $\mathrm{M}$. Ambroise Dupont, Sénateurs.
}

\footnotetext{
${ }^{1}$ Cet article a bénéficié du soutien du programme "Atouts pour publier" dispensé par la FNEGE en 2010.

2 L'auteur est à ce jour Maître de Conférences des
Universités en Sciences de Gestion à l'Institut d'Administration des Entreprises (IAE) de SaintEtienne, qu'il dirige, et membre du laboratoire COACTIS (EA 4161)

Adresse postale : 2, rue Tréfilerie 42023 SAINTETIENNE CEDEX 2
} 


\section{Politique contractuelle et stratégies universitaires : le rendez-vous manqué ?/ Hervé GOY}

s'imposer comme levier efficace des stratégies universitaires en France ? Si, depuis près d'un demi-siècle, l'enseignement supérieur fait l'objet de multiples recherches dans des champs disciplinaires variés (éducation, sociologie, économie, science politique...), c'est à travers notre regard de stratégiste, posé sur la trajectoire singulière d'une université, que nous aborderons cette problématique. Plus spécifiquement, et d'un certain point de vue dans le prolongement des travaux de Musselin (1997), la stratégie de cette université sera envisagée sous l'angle de l'examen du contenu et des conditions d'élaboration de cinq contrats d'établissement couvrant la période 1991-2010. Notre texte sera organisé en trois parties. Nous dresserons, pour commencer, un panorama synthétique des travaux académiques qui questionnent la pertinence du rapport entre stratégie et université. Ce faisant, nous déroulerons dans une deuxième partie méthodologique la présentation de I'université étudiée, ainsi que la description des modalités d'examen des trois matériaux empiriques retenus. Après quoi, nous présenterons dans une troisième partie trois propositions en réponse à la problématique soulevée dans cet article. Enfin, une conclusion nous permettra d'évoquer les limites, implications et prolongements à notre travail.

\section{Cadre théorique}

Du point de vue de certains courants de pensée, compte tenu de leurs modes traditionnels de fonctionnement ou selon les principes organisationnels qui les régissent, les universités ne sont généralement pas décrites, dans la littérature académique, comme les organisations qui se prêtent le mieux à la pratique de la stratégie. L'analyse, par certains auteurs, de l'évolution récente des systèmes

\footnotetext{
${ }^{4}$ Citation tirée d'un texte rédigé par Bernard Dizambourg sur les spécificités de la stratégie des universités :

http://www.esen.education.fr/fileadmin/user upl oad/Modules/Ressources/Conferences/flash/0910/strategie-mesr/medias/strategiemesr dizambourg b.pdf
}

d'enseignement supérieur internationaux vient néanmoins contrebalancer ce constat.

\subsection{Du (difficile) mariage entre stratégie et universités}

La stratégie (en tant que domaine disciplinaire) n'est pas simple à appréhender sur le champ universitaire, pour de multiples raisons. En particulier pour B. Dizambourg", "I'une des principales difficultés dans le cas des universités est que celles-ci (en particulier les universités à champs disciplinaires larges) évoluent sur des environnements de recherche et de formation dont les caractéristiques stratégiques (quelles sont les conditions de succès, que faut-il maitriser, quel est l'environnement géographique pertinent : régional, national, européen, mondial) ne sont pas homogènes". Plus globalement, c'est souvent la question de la transposition des principes traditionnels de la stratégie aux secteurs publics qui est soulevée. Bonaccorsi et Daraio (2007) ou Buckland (2009) s'accordent ainsi à dire que la notion de stratégie, telle qu'envisagée dans le "monde des affaires" ${ }^{15}$, reste difficilement transposable dans la plupart des secteurs publics, en particulier l'enseignement supérieur. II serait d'après eux inapproprié de parler de stratégie au sujet des universités publiques, prisonnières de la "cage de fer" (au sens wébérien) de leurs origines historiques, et soumises à des règles institutionnelles qui limitent fortement le périmètre de tout comportement discrétionnaire (en un mot toute stratégie). Une telle vision s'enracine très clairement dans la sociologie néoinstitutionnaliste issue des travaux fondateurs de Meyer et Rowan (1977), et s'inscrit à notre sens dans le prolongement des écrits de DiMaggio et Powell (1983) qui ont distingué trois principaux mécanismes d'isomorphisme

\footnotetext{
${ }^{5}$ C'est-à-dire fondée sur les principes traditionnels de la planification stratégique (en termes de mission, vision, intention stratégique, analyse de l'environnement, buts et objectifs, mise en œuvre, contrôle...), étant entendu que l'expression "monde des affaires" ne peut en aucun cas désigner selon nous une réalité univoque.
} 
institutionnel (coercitif, normatif et mimétique). De surcroit au sens de la théorie de la dépendance des ressources (Pfeffer, Salancik, 1978), la reconnaissance d'une capacité stratégique est liée à l'aptitude d'une organisation de lever une partie substantielle de ses ressources. Or, en tant qu'institutions publiques (particulièrement en Europe continentale), les universités ne peuvent s'endetter sur les marchés financiers, et bénéficient de dotations limitées issues pour l'essentiel de fonds publics (gouvernementaux ou territoriaux). Pour Chevaillier (2002), cette absence d'autonomie des universités dans la collecte de ressources (qui leur permette d'engager un processus d'allocation cohérent avec les objectifs qu'elles s'assignent) constitue clairement un frein au développement d'une stratégie.

En second lieu, il convient d'évoquer l'effet modérateur de deux principes régisseurs des systèmes d'enseignement supérieur publics sur la capacité stratégique des universités : la dépendance à l'égard de l'Etat d'une part ; le degré de participation des acteurs dans l'organisation et le fonctionnement de leurs universités d'autre part. Pour Mallet, Balme et Richard (2002), ces deux principes sont très marqués dans le cas français : d'une part, la participation des acteurs universitaires à l'organisation et au fonctionnement de leur établissement est mentionnée de façon constante dans les textes de loi depuis 1968 ; d'autre part, la dépendance des établissements d'enseignement supérieur à l'égard de l'Etat se traduit, en particulier, par le statut de fonctionnaire de l'Etat des personnels, "le plus puissant des instruments par lesquels l'Etat garantit la dépendance de l'établissement d'enseignement public à son égard ". Historiquement, la coexistence de ces deux principes dans le fonctionnement du système d'enseignement supérieur public français a clairement obéré les capacités stratégiques des universités, au bénéfice $d u$ pouvoir exercé par les universitaires euxmêmes (dans le cadre de la défense de logiques disciplinaires et/ou facultaires), et sous le contrôle étroit de la tutelle publique. Sur ce plan, force est de constater toutefois qu'avec l'introduction de la politique contractuelle au tournant des années 19801990, " c'est tout le système universitaire français qui s'en est trouvé ébranlé et redéfini» (Musselin, 2001).

La plupart de ces travaux nous renvoie de facto à la question de l'(in)adaptation des configurations organisationnelles universitaires aux cadres d'analyse de la stratégie. Des auteurs de référence en management se sont en effet intéressés, dans le courant des années 1970, aux particularités du fonctionnement universitaire pour élaborer des cadres d'analyse aujourd'hui largement diffusés. C'est ainsi que Cohen et March (1974) ont défini les principes de "l'anarchie organisée" : des organisations sans objectifs vraiment cohérents et partagés, où le processus de production peu matériel relève d'une technologie complexe, où la majeure partie du personnel exerce une "pratique privée" d'intervention directement auprès des "clients" de l'organisation sans qu'il soit possible et réaliste d'assumer une supervision constante des tâches effectuées, et dont les membres participent de façon intermittente et plus ou moins active, voire vraiment intéressée, aux différentes prises de décision qui affectent l'ensemble de l'organisation. Sur un autre registre, c'est en étudiant les organisations éducatives que Weick (1976) a défini la notion de "systèmes faiblement liés", dont les particularités tiennent à l'indépendance de sous-unités dans la poursuite de leurs objectifs propres, ainsi qu'à des processus de décision autonomes et autorégulés. En conséquence de quoi pour Bartoli (2002), la référence au modèle de la "bureaucratie professionnelle" de Mintzberg (1982) pour qualifier les organisations universitaires est devenue incontournable : le centre opérationnel de l'université est composé des spécialistes de chaque discipline, qui ont amplement le contrôle de leur propre activité dont ils sont, pour l'essentiel, les seuls responsables. Le sommet stratégique n'ayant que peu de prérogatives directes sur ceux-ci, il exerce en revanche pleinement son autorité sur le centre logistique (constitué des personnels administratifs et techniques), 


\section{Politique contractuelle et stratégies universitaires : le rendez-vous manqué ?/ Hervé GOY}

tandis que la technostructure (l'entité chargée du soutien, de la programmation et de la normalisation des processus) n'existe presque pas. Barth (2012) va même jusqu'à présenter I'université française comme une structure où se côtoient des clans, ayant comme objectifs particuliers la préservation de leurs intérêts et de leurs périmètres avec en commun le mot d'ordre tacite de la perpétuation du système et de sa stabilité, au nom de l'excellence académique.

A première vue, ces différentes perspectives théoriques pourraient suffire à expliquer pourquoi la contractualisation peine à s'imposer comme levier efficace des stratégies universitaires en France : la notion même de stratégie universitaire semble sujette à caution ! Un autre regard sur l'évolution récente des systèmes d'enseignement supérieur invite cependant à approfondir le sujet.

\subsection{L'avènement de l'université entreprenante}

D'après le rapport $2009^{6}$ de la conférence mondiale de I'UNESCO sur l'enseignement supérieur, les transformations intervenues à la fin du $20^{\text {ème }}$ siècle et au début du $21^{\text {ème }}$ siècle ont été « d'une ampleur et d'une diversité sans précédent »: la mondialisation aurait déjà intensément influencé l'enseignement supérieur, devenu clairement une activité concurrentielle. Dans le courant des années 1990, de nombreux auteurs constataient déjà la profondeur des changements intervenus dans les systèmes universitaires internationaux. A titre d'illustration pour Clark (1998), "les universités du monde entier vivent une époque d'instabilité inquiétante, caractérisée par l'accroissement du nombre d'étudiants et de leur diversité, la pression du marché du travail liée au développement des

\footnotetext{
${ }^{6}$ Altbach P.G., L. Reisberg et L.E. Rumbley, Trends in global higher education: tracking an academic revolution

${ }^{7}$ Kirby et al. (2011) ont listé pas moins d'une dizaine de définitions contradictoires dans la littérature académique : compte tenu de la « nébuleuse » qui
}

activités économiques basées sur les connaissances, le désengagement financier des états, la difficile maîtrise de la croissance de la production des savoirs ". Dans le même temps, des changements dans la culture organisationnelle des universités semblent être intervenus : McNay (1995) a notamment mis en évidence la montée en puissance de la culture d'entreprise (au sens du développement d'aptitudes et de compétences issues du "monde des affaires") au sein même des établissements d'enseignement supérieur tout au long de la décennie 1990. La conjonction de ces deux facteurs expliquerait, dès lors, la promotion d'une nouvelle forme d'organisation : l'université entrepreneuriale.

Chercher à caractériser la dimension entrepreneuriale d'une université n'est pas chose aisée (Goy, 2013). En rupture avec les visions antérieures des modes d'organisation universitaire (à dominante tantôt collégiale et auto-organisée, politique et conflictuelle ou symbolique et peu directive), la perspective de l'université entrepreneuriale se présente avant tout comme une réponse aux enjeux universitaires contemporains. Cette capacité des universités à développer un "modèle entrepreneurial" ne va cependant pas de soi : Askling et Kristensen (2000) ou Davies (2001) ont en effet souligné que l'adoption par les universités d'une forme organisationnelle plus entrepreneuriale dépend avant tout de leur capacité à développer des stratégies, à mesurer leur niveau de performance, à diminuer leurs coûts, à évaluer la qualité de leurs activités d'enseignement et de recherche, à développer des partenariats externes, à renforcer leur direction institutionnelle... Au demeurant pour Clark (1998) comme pour Etzkowitz et al. (2000)', l'évolution rapide des caractéristiques des systèmes d'enseignement supérieur (un

règne entre les notions d'entrepreneuriat universitaire, de capitalisme académique, d'université-entreprise ou bien encore d'université entrepreneuriale, c'est précisément l'acception commune aux travaux de Burton Clark et Henry Etzkowitz que nous retiendrons dans cet article. 
déséquilibre croissant entre les demandes adressées aux universités et leurs aptitudes à y répondre tout en conservant leur forme traditionnelle) oblige les universités de nombreux pays à devenir davantage entreprenantes. En ce sens, les universités entreprenantes sont celles qui cherchent à exploiter leurs possibilités au-delà de leurs moyens disponibles, et qui prennent le risque de revisiter leur organisation comme leurs modes de réaction aux pressions internes ou externes qu'elles subissent. Cette dernière perspective se rapproche selon nous de l'une des définitions de référence de la stratégie formulée par Tabatoni et Jarniou (1975), envisagée sous l'angle des modes relationnels avec l'environnement externe comme à l'intérieur de l'organisation : en d'autres termes, les universités contemporaines (devenues entreprenantes) n'auraient d'autre choix que de développer des stratégies, point sur lequel Mailhot et Schaeffer (2009) ont encore récemment insisté.

II est intéressant de noter que le développement de cette vision entrepreneuriale de l'organisation des universités coïncide assez bien sur le plan chronologique, sous l'impulsion des politiques publiques, avec la mise en œuvre de dispositifs managériaux (inspirés des méthodes traditionnelles de planification) dans la plupart des systèmes d'enseignement supérieur, notamment en Europe. C'est à partir de 1992 que des plans stratégiques (précisant les buts et objectifs académiques, le nombre d'étudiants visés, les personnels nécessaires, les ressources physiques et pédagogiques, les moyens financiers et la mesure de la qualité des activités) ont, par exemple, été formellement requis pour l'ensemble des institutions d'enseignement supérieur $\mathrm{du}$ Royaume-Uni (Bargh et al., 2000). De même en France, les premiers contrats d'établissement

\footnotetext{
8 En application de la Circulaire n89-079 du 24 mars 1989 relative à la politique contractuelle liant l'Etat et les universités. Nous renvoyons par ailleurs le lecteur au chapitre 5 de l'ouvrage de Musselin (2001) pour un aperçu historique de la mise en œuvre de la contractualisation en France.
}

entre les universités et leur tutelle, visant à spécifier la politique de chacune en matière de formation, de recherche et de pilotage pour quatre années, furent signés dès le début des années $1990^{8}$. A travers ce dispositif, trois objectifs étaient particulièrement visés : la contractualisation de l'attribution d'une partie des ressources étatiques; le renforcement de l'autonomie des établissements ; la formation d'une identité institutionnelle autour d'objectifs explicités9.

D'après cette littérature, le tournant entrepreneurial entamé par les universités depuis une vingtaine d'années devrait donc les avoir conduit, plus que par le passé, à développer des stratégies singulières. Au-delà de "l'invariable soubassement normatif " de ces travaux (tel que dénoncé par MignotGérard, 2003), force est de constater que le rapport cité en introduction de cet article n'aboutit pas à cette conclusion. Les choix méthodologiques retenus pour en comprendre les raisons sont maintenant précisés.

\footnotetext{
${ }^{9}$ Consulter pour de plus amples développements le rapport d'évaluation de la politique de contractualisation avec les universités réalisé en 2003 par Technopolis France, cabinet de conseil spécialisé dans l'analyse et l'évaluation des politiques publiques.
} 


\section{Méthodologie}

Trois types de matériaux ont été mobilisé dans le cadre de cette recherche : le recueil d'entretiens avec des acteurs universitaires en charge de la direction de leur établissement sur l'ensemble de la période couverte; le contenu proprement dit des contrats d'établissements ; les documents d'orientation transmis par le ministère aux présidents d'universités préalablement au démarrage de chaque processus de contractualisation. Avant de les détailler, nous commencerons par présenter les raisons $\mathrm{du}$ choix de notre champ d'investigation.

\subsection{Une université pluridisciplinaire de taille moyenne pour terrain de recherche}

C'est au début des années 1960 que se sont développées en terres ligériennes les premières filières d'enseignement supérieur universitaire, entraînant la création de I'Université Jean Monnet - Saint-Etienne (UJM) en 1969. Ce "jeune" établissement fait, à ce jour, partie des 21 universités françaises dites "pluridisciplinaires avec santé" ${ }^{10}$, et accueille près de $16^{\circ} 000$ étudiants répartis en cinq grands domaines (droit - économie - gestion ; arts - lettres - langues ; sciences humaines et sociales ; sciences, technologie et ingénierie ; santé). Les activités de I'UJM se répartissent sur cinq campus géographiques distincts, majoritairement à Saint-Etienne mais aussi à Roanne.

Le choix de cette université, à laquelle nous sommes institutionnellement rattachés, tient tout d'abord à un accès plus aisé aux données d'enquête. Cette remarque vaut tout autant

\footnotetext{
10 En référence à la catégorisation traditionnelle opérée jusqu'à présent par le Ministère de l’Enseignement Supérieur et de la Recherche, qui distingue par ailleurs les universités de "sciences et/ou santé", "pluridisciplinaires hors santé", "lettres - sciences humaines" et "droit - économie - gestion".

11 Caractéristique encore soulignée dans la lettre d'échange relative au projet de contrat quadriennal
}

pour les textes des contrats proprement dits que pour la possibilité d'entrer en contact avec les principaux décideurs sur une période de vingt années. En second lieu, l'UJM est traditionnellement considérée par sa tutelle publique comme l'une des universités médianes de référence dans le système français ${ }^{11}$. Si cette caractéristique ne permet en aucun cas de généraliser nos propos à l'ensemble des universités, elle exprime néanmoins l'idée selon laquelle les caractéristiques de I'UJM (en termes de dimensionnement, de population étudiante, de champs disciplinaires,...) en font un terrain d'observation propice des principales évolutions qui interviennent dans l'enseignement supérieur français. La trajectoire de cette université, revisitée sur la période 1991-2010 à l'aune du texte de ses cinq contrats, est succinctement présentée en annexe 1.

\subsection{Présentation des matériaux empiriques analysés}

Comme le souligne le rapport précité (cf. note 8), la préparation d'un contrat d'établissement commence environ deux ans avant le début de la période contractualisée, et s'opère à partir de la formulation d'un projet de développement par l'établissement ${ }^{12}$. Du côté de l'université, la préparation du projet se fait sous la responsabilité du Président qui s'appuie le plus souvent sur les instances décisionnelles ou propositionnelles (comme les conseils centraux ou le bureau), et éventuellement sur des groupes de travail. Du côté ministériel, plusieurs directions sont traditionnellement impliquées dans la contractualisation avec les universités, avec l'appui des conseillers d'établissements qui

du 6 novembre 2006 : " Les axes définis doivent conforter l'identité de l'université, analysée comme un établissement médian qui doit renforcer (...) ".

12 Le processus décrit dans cette section valait pour les contrats signés entre chaque université et sa tutelle ministérielle antérieurement à la mise en œuvre de la loi du 22 juillet 2013. 
jouent un rôle d'intermédiaire entre l'administration centrale et les établissements, mais aussi entre les différentes directions au sein même du ministère. Le rôle de ce dernier est, d'une part, d'informer les présidents d'universités sur les orientations de l'Etat en matière de politique d'enseignement supérieur et de recherche et sur ses attentes vis-à-vis des universités au début de la préparation du contrat et, d'autre part, de réceptionner ensuite les projets d'établissements avec leurs annexes. Les dossiers à évaluer sont ensuite distribués aux instances d'évaluation, dont les conclusions permettent à la direction de l'enseignement supérieur de se forger un avis sur l'établissement dans son ensemble, en s'appuyant sur le projet de développement de l'établissement, les évaluations et une visite in situ. Après cette visite, le ministère renvoie à l'université une proposition du contrat, qui repose sur le projet d'établissement assorti de modifications et/ou suggestions. Ce n'est que lorsque le texte du contrat est accepté par les deux parties que commence la négociation financière, qui aboutit à la signature formelle du contrat.

- Matériau $n^{\circ} 1$ : le contexte perçu au moment de l'élaboration des projets d'établissement

La collecte de données relatives aux conditions d'élaboration des cinq projets d'établissement de l'UJM a été réalisée par la conduite de trois entretiens semi-directifs ${ }^{13}$, auprès des trois anciens présidents de I'UJM ayant occupé, en outre, des fonctions centrales ${ }^{14}$ dans les quatre équipes de direction qui se sont succédées à la tête de l'UJM entre début 1987 et fin 2006. Plusieurs catégories de données ont ainsi pu être recueillies pour chaque campagne de contractualisation : la perception par les intéressés du contexte

\footnotetext{
13 D'une durée de 30,70 et 80 minutes selon la disponibilité des intéressés.

${ }^{14}$ Vice-président du Conseil d'Administration ; Viceprésident du Conseil Scientifique ; Vice-président chargé des Finances.
}

national puis local dans lesquels s'inscrivait la préparation de chaque contrat, ainsi que le dispositif interne à l'UJM mis en œuvre pour leur élaboration.

Matériau $n^{\circ} 2$ : le contenu des contrats d'établissement de l'UJM entre 1991 et 2010

Partant de l'idée selon laquelle le contenu d'un contrat d'établissement peut s'apparenter au résultat d'une alchimie entre l'histoire de l'université, le dispositif mis en œuvre pour le préparer et l'implication des responsables universitaires (Musselin, 1997), nous avons également procédé à l'analyse qualitative des textes des cinq contrats de I'UJM. La codification thématique a été réalisée à l'aide du logiciel d'analyse de discours NVivo (8), à partir de six catégories de codage issues de la transposition, sur le champ universitaire, de la "définition implicite" du domaine de la stratégie formulée par Nag, Hambrick et Chen $(2007)^{15}$ : nous avons ainsi appréhendé le management stratégique universitaire sous I'angle des orientations et finalités définies par l'équipe de direction, conduisant à mobiliser des ressources en vue d'accroître la performance de l'université dans son environnement externe. Le tableau 1 ci-après présente la retranscription de ces six catégories dans le logiciel NVivo.

\footnotetext{
15 "The field of strategic management deals with (a) the major intended and emergent initiatives (b) taken by general managers on behalf of owners, (c) involving utilization of resources (d) to enhance the performance (e) of firms (f) in their external environments".
} 
Tableau 1 : L'arborescence thématique de l'analyse textuelle des contrats de l'UJM

\begin{tabular}{|c|c|c|c|}
\hline Type & Name & Memo Link & Sources \\
\hline Tree Node & \multicolumn{2}{|l|}{ STRATEGIE } & \\
\hline & \multirow{5}{*}{ Tree Node } & \multicolumn{2}{|c|}{ MR - mobilisation de ressources } \\
\hline & & Tree Node & MR - pilotage \\
\hline & & Tree Node & MR - recherche \\
\hline & & Tree Node & MR - formations \\
\hline & & Tree Node & MR - infrastructures \\
\hline & \multirow{4}{*}{ Tree Node } & UN - université & \\
\hline & & Tree Node & UN - identité \\
\hline & & Tree Node & UN - structuration \\
\hline & & Tree Node & UN - engagements \\
\hline & \multirow[t]{6}{*}{ Tree Node } & OF - orientations \& finalités & \\
\hline & & Tree Node & OF - pilotage \\
\hline & & Tree Node & OF - recherche \\
\hline & & Tree Node & OF - formations \\
\hline & & Tree Node & OF - international \\
\hline & & Tree Node & OF - structure \\
\hline & \multirow{5}{*}{$\begin{array}{l}\text { Tree Node } \\
\text { Tree Node }\end{array}$} & ED - équipe de direction & \\
\hline & & PE - performance & \\
\hline & & Tree Node & PE - pilotage \\
\hline & & Tree Node & $\mathrm{PE}$ - recherche \\
\hline & & Tree Node & PE - formations \\
\hline & \multirow{3}{*}{ Tree Node } & EE - environnement externe & \\
\hline & & Tree Node & EE - concurrence \\
\hline & & Tree Node & EE - réseaux \\
\hline
\end{tabular}

- Matériau $n^{\circ} 3$ : les documents d'orientation transmis par le ministère à la présidence de l'UJM

Préalablement au démarrage de chaque processus de contractualisation, la direction en charge de l'enseignement supérieur ${ }^{16} \mathrm{du}$ ministère concerné ${ }^{17}$ communique à chaque Président d'université de la vague contractuelle correspondante (vague A pour I'UJM) des éléments d'orientation. Ce cadrage

16 Successivement « Direction de l'Enseignement Supérieur " (DES), « Direction Générale de I’Enseignement Supérieur » (DGES) puis Direction Générale de l'Enseignement Supérieur et de I'Insertion Professionnelle (DGESIP) sur la période couverte par notre analyse. a pris des formes variables d'un contrat à l'autre s'agissant de l'UJM :

- circulaire d’orientations n98-118 du 22-51998 publiée au bulletin officiel du 4 juin 1998 annonçant la nature, la méthode et les finalités de la campagne de contractualisation 1999/2002 ;

- courriers du 4 juillet et du 12 octobre 2001 précisant la méthode et les objectifs de la campagne de contractualisation 2003/2006 ;

17 Ministère de l'Education Nationale incluant le périmètre de l'ESR ou Ministère de l'Enseignement Supérieur et de la Recherche " de plein exercice " selon les alternances politiques. 
- courriers du 20 juin 2005 et du 6 novembre 2006 relatifs au mode d'emploi et à la lettre d'échanges de la campagne de contractualisation 2007/2010.

Sur ce troisième volet, il s'agira pour nous de mettre en correspondance les orientations contenues dans chacun de ces documents avec le texte final des contrats correspondants $^{18}$.

Pourquoi la contractualisation n'a-t-elle pas réussi à s'imposer comme levier efficace des stratégies universitaires en France ? Le traitement de l'ensemble de ces matériaux empiriques nous permet de formuler trois propositions en réponse à notre problématique, présentées ci-après.

\section{Résultats}

Nous présenterons successivement notre analyse du contexte au démarrage de chacune des phases de contractualisation, du contenu thématique de chaque contrat de l'UJM, ainsi que de leur lien avec les documents d'orientation transmis par le ministère.

\subsection{Le contexte de la politique contractuelle : des contrats pour quoi faire?}

Dans quelles conditions (nationales comme locales) la préparation de chacun des projets d'établissement de I'UJM s'inscrivait-elle ? Quels types de processus internes leur élaboration a-t-elle suivie ? La restitution synthétique de ces informations, collectées à l'occasion des entretiens menés auprès de trois des anciens présidents de l'université, est présentée dans le tableau 2 suivant.

18 La consultation des archives de la Direction de l'université ne nous ayant pas permis de remonter plus en amont dans le temps, cette partie de notre travail ne portera donc que sur ces trois contrats de I'UJM. 
Tableau 2 : Une vue synthétique des conditions d'élaboration des cinq contrats de l'UJM

\begin{tabular}{|c|c|c|c|c|}
\hline Contrat & Nom / fonction & Perception du contexte NATIONAL & Perception du contexte LOCAL & Processus d'élaboration du CONTRAT \\
\hline 1991-1994 & $\begin{array}{l}\text { B.E. (1987-1991) } \\
\text { Vice-président du } \\
\text { Cons. d'Administration } \\
\text { M.V. (1992-1996) } \\
\text { Vice-président chargé } \\
\text { des Finances }\end{array}$ & $\begin{array}{l}\text { Périmètre de la recherche maintenu en dehors de la } \\
\text { politique contractuelle par la tutelle } \\
\text { Phase de développement de l'enseignement supérieur } \\
\text { engendrant un "enthousiasme général" } \\
\text { Engagement fort des conseillers d'établissement } \\
\text { auprès des universités }\end{array}$ & $\begin{array}{l}\text { Mobilisation des acteurs ("effervescence des esprits") en } \\
\text { lien avec le contexte national } \\
\text { Une équipe de direction soudée, composée de collègues } \\
\text { présents depuis la création de l'université }\end{array}$ & $\begin{array}{l}\text { Rôle essentiel des composantes (difficulté de mise en cohérence des } \\
\text { initiatives par la présidence) } \\
\text { Logique participative dans le processus de préparation } \\
\text { Visée du contrat = obtenir des moyens supplémentaires }\end{array}$ \\
\hline $1995-1998$ & $\begin{array}{l}\text { B.E. (1992-1996) } \\
\text { Président } \\
\text { M.V. (1992-1996) } \\
\text { Vice-président chargé } \\
\text { des Finances } \\
\text { M.v. (1997-2001) } \\
\text { Président }\end{array}$ & $\begin{array}{l}\text { Alternance politique en cours de préparation du } \\
\text { contrat (rigueur sur les moyens ; pas de réelle } \\
\text { négociation politique avec la tutelle) } \\
\text { Enjeu principal sur les habilitations de diplômes } \\
\text { Question des emplois sortie du processus contractuel } \\
\text { par la tutelle } \\
\text { Périmètre de la recherche toujours absent de la } \\
\text { politique contractuelle d'établissement } \\
\text { Volonté de couper court aux négociations isolées par } \\
\text { un affichage fort du rôle de l'université dans la lettre de } \\
\text { cadrage ministérielle } \\
\text { Souci de la tutelle d'harmoniser les cartes de formation } \\
\text { régionales }\end{array}$ & $\begin{array}{l}\text { Croissance des effectifs étudiants (difficultés } \\
\text { d'encadrement + pénurie de locaux) } \\
\text { Pas de préoccupation sur la stratégie à moyen et long } \\
\text { terme (enjeu = réponse satisfaisante à la demande) } \\
3 \text { attentes vis-à-vis du contrat : création de postes, } \\
\text { habilitations de diplômes nouveaux et hausse de la } \\
\text { dotation financière pour faire face aux charges } \\
\text { d'enseignement } \\
\text { Adhésion forte et naturelle des acteurs internes au } \\
\text { processus (place prépondérante des thèmes } \\
\text { pédagogiques): importante adhésion au processus (le } \\
\text { contrat perçu comme évolution porteuse) }\end{array}$ & $\begin{array}{l}\text { Formalisation d'une lettre de cadrage du Président préparée en } \\
\text { Bureau } \\
\text { Définition de } 3 \text { pôles structurants d'identification de l'UJM en fin de } \\
\text { processus } \\
\text { Démarche ascendante à partir des composantes (rôle essentiel des } \\
\text { doyens dans la préparation du contrat) } \\
\text { Faible implication du Président dans la synthèse (forte délégation aux } \\
\text { Vice-présidents CA et surtout CEVU) } \\
\text { Arbitrages des projets remontés des composantes par le conseil } \\
\text { d'administration (choix des axes, rédaction de la politique générale et } \\
\text { de la demande de moyens) } \\
\text { La tutelle comme cible évidente du contrat (pas de visée } \\
\text { instrumentale en interne car la question ne se posait pas) }\end{array}$ \\
\hline 1999-2002 & $\begin{array}{l}\text { M.V. (1997-2001) } \\
\text { Président } \\
\text { R.F. (1997-2001) } \\
\text { Vice-président du } \\
\text { Cons. d'Administration }\end{array}$ & $\begin{array}{l}\text { Impulsion en } 1997 \text { (alternance politique) de la politique } \\
\text { contractuelle (hausse de la part contractuelle dans } \\
\text { l'attribution des moyens) } \\
\text { Intégration du périmètre de la recherche dans la } \\
\text { démarche de contractualisation } \\
\text { Rôle moindre des conseillers d'établissement dans la } \\
\text { préparation - négociation des contrats }\end{array}$ & $\begin{array}{l}\text { Concomitance entre contractualisation } 1999-2002 \text { et } \\
\text { préparation du CPER } 2000-2007 \text { (affirmation identitaire } \\
\text { par la structuration de la recherche) } \\
\text { Volonté affichée de séparer la fonction administrative du } \\
\text { rôle politique } \\
\text { Renversement de tendance des effectifs (seuil des } \\
15^{\circ} 000 \text { étudiants atteint en } 1998-1999 \text { ) } \\
\text { Forte demande de moyens (postes, surfaces ...) }\end{array}$ & $\begin{array}{l}\text { Forte dynamisation du processus interne de préparation compte tenu } \\
\text { des perspectives de retombées financières } \\
\text { Le contrat d'établissement comme consolidation des initiatives des } \\
\text { composantes (logique ascendante prédominante) } \\
\text { Négociation interne entre directeurs de composantes et équipe de } \\
\text { direction pour apparaître "en bonne place" dans le contrat }\end{array}$ \\
\hline 2003-2006 & $\begin{array}{l}\text { R.F. (2002-2006) } \\
\text { Président }\end{array}$ & $\begin{array}{l}\text { Changement de Directeur Général au Ministère } \\
\text { Des attentes de la tutelle en termes d'affichage } \\
\text { synthétique des orientations de l'établissement } \\
\text { La volonté de diminuer le nombre de lignes } \\
\text { d'attribution de moyens } \\
\text { Des interlocuteurs plus "techniciens" que "politiques" } \\
\text { au niveau de la tutelle }\end{array}$ & $\begin{array}{l}\text { Le contrat encore largement envisagé comme manne } \\
\text { financière potentielle par la direction de l'université } \\
\text { comme par les acteurs de base } \\
\text { Passage de relai naturel entre M.V. et R.F. dans la } \\
\text { transition entre les contrats } 1999-2002 \text { et } 2003-2006\end{array}$ & $\begin{array}{l}\text { Une place plus importante des services centraux et/ou communs dans } \\
\text { le processus de conception } \\
\text { Des composantes et services consultés pour identifier les lignes } \\
\text { directrices du contrat (pas de projets spécifiques) } \\
\text { Synthèse et propositions par l'équipe de direction en réunion } \\
\text { mensuelle des directeurs puis en CA } \\
\text { Rôle plutôt stratégique du CS et plutôt académique du CEVU }\end{array}$ \\
\hline $2007-2010$ & $\begin{array}{l}\text { B.E. (2002-2006) } \\
\text { Vice-président du Conseil } \\
\text { Scientifique } \\
\text { R.F. (2002-2006) } \\
\text { Président }\end{array}$ & $\begin{array}{l}\text { "Evolution culturelle" de la tutelle : impulsions fortes } \\
\text { sur des logiques de projets, d'indicateurs et } \\
\text { d'engagement (impact de la LOLF + prémisses de } \\
\text { l'autonomie) }\end{array}$ & $\begin{array}{l}\text { Evolution sensible de la vision du contrat par l'équipe de } \\
\text { direction : le contrat comme moyen de se donner des } \\
\text { horizons et des objectifs partagés collectivement }\end{array}$ & $\begin{array}{l}\text { Distanciation marquée du couple Président/Secrétaire Général dans } \\
\text { la préparation du contrat } \\
\text { Très forte implication (personnalisation) du Président dans la } \\
\text { conception du contrat }\end{array}$ \\
\hline
\end{tabular}


Ainsi pouvons-nous relever l'effet d'un certain nombre d'éléments contextuels sur le développement de la stratégie de I'UJM entre 1991 et 2010. On notera, tout d'abord, la prise en main progressive de la structuration du processus interne d'élaboration des contrats par les présidents et leurs équipes : si la préparation des trois premiers contrats a surtout consisté, pour la direction de l'UJM, à réaliser la synthèse des actions élaborées au niveau des composantes, la présidence a résolument prédéfini les thèmes directeurs des deux derniers contrats. On soulignera, ensuite, l'importance accordée par I'UJM à l'affirmation de son identité, en particulier par la structuration de sa politique scientifique depuis la fin de années 1990, qui semble tenir tout autant à l'intégration du périmètre de la recherche à la politique contractuelle par le Ministère, qu'à la nécessité de trouver un positionnement lisible dans son environnement régional, dans le contexte de préparation du contrat de plan état-région qui s'amorçait. On précisera, enfin, que si la trajectoire stratégique de I'UJM peut être lue à travers la dynamique interne de ses activités (la variation du nombre d'étudiants qu'elle accueille s'agissant des orientations des deux premiers contrats), la tension progressive créée par les conditions de sa nécessaire inscription dans un environnement perçu comme plus menaçant joue également un rôle important dans les orientations de ses deux derniers contrats. Un point commun transparaît néanmoins largement en ce qui concerne les finalités perçues du contrat d'établissement par les acteurs de I'UJM, ce qui nous conduit à formuler une première proposition : la contractualisation n'a pas réussi à s'imposer comme levier efficace des stratégies universitaires, parce qu'elle a essentiellement été envisagée comme vecteur pour obtenir des ressources supplémentaires. A l'exception notable de la préparation du contrat 2007-2010 (signe révélateur de l'amorce d'un changement ?), la mobilisation du processus contractuel pour construire des horizons et des objectifs partagés collectivement est en effet très largement absente des pratiques de I'UJM telles qu'analysées. Au demeurant, un examen plus approfondi du contenu de chacun des contrats de I'UJM va nous permettre de compléter ce premier constat.

\subsection{Le contenu des contrats : parle-t-on vraiment de choix stratégiques ?}

La dimension stratégique qui ressort le plus à la lecture des contrats de I'UJM correspond à la recherche de coopérations et au développement de partenariats (cf. annexe 1). En stratégie des organisations, il convient toutefois de distinguer les choix stratégiques (stratégies de coûts, différenciation, diversification, spécialisation...) des modalités de développement (croissance interne, fusions, acquisitions, alliances, partenariats...) qui permettent de les mener à bien (Johnson, Whittington, Scholes, Fréry, 2011). II se trouve que si les coopérations et partenariats relèvent très clairement de la seconde catégorie, au service de quels choix stratégiques œuvrent-ils pour I'UJM ? Les résultats d'une analyse longitudinale $\mathrm{du}$ contenu de ses cinq contrats d'établissement, selon une double lecture (par période et par thème), permet d'apporter un éclairage sur ce point. 


\section{Politique contractuelle et stratégies universitaires : le rendez-vous manqué ?/ Hervé GOY}

Figure 1 : Le profil thématique du contenu stratégique des contrats de l'UJM

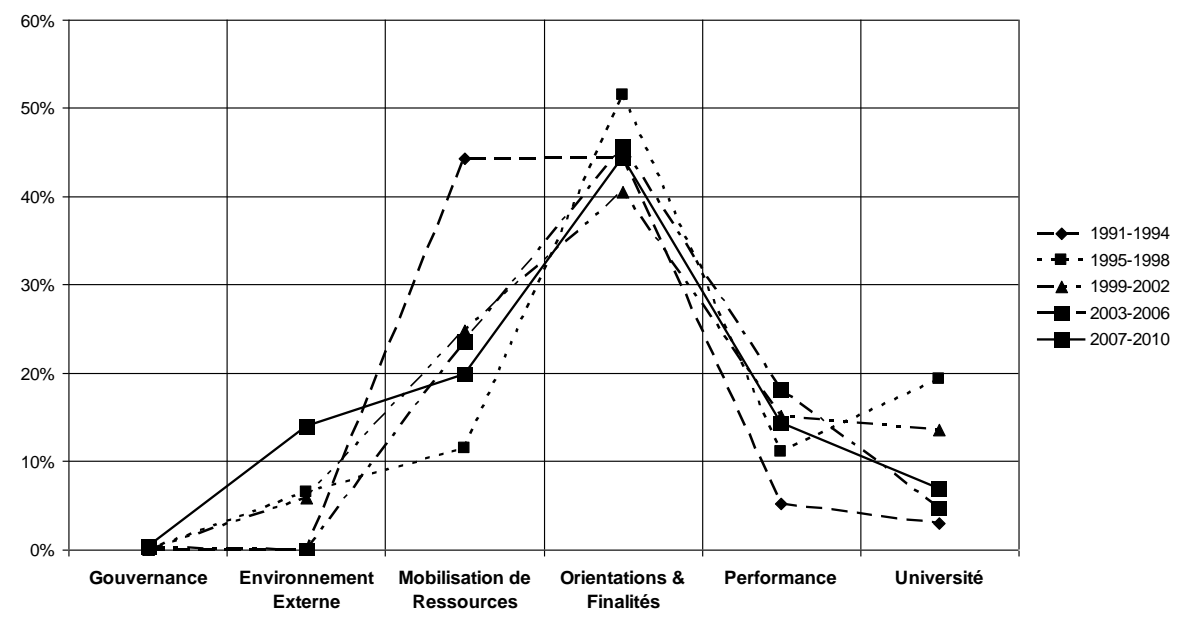

Au regard de la grille de lecture mobilisée (Nag, Hambrick, Chen, 2007), on peut noter la tendance commune que le contenu des cinq contrats d'établissement suit : l'item stratégique le plus fréquemment évoqué dans les textes analysés renvoie aux orientations et finalités données aux différentes missions (enseignement, recherche, valorisation) de I'UJM. Vient ensuite la question de la mobilisation des ressources (humaines, financières et organisationnelles), puis la thématique de la performance (en termes d'attractivité des étudiants et de visibilité des activités de recherche notamment). Les deux catégories de mots-clés les moins évoquées concernent la référence à l'université en tant qu'acteur institutionnel pour l'une, et au rôle comme à la place de l'équipe de direction pour l'autre.

Ainsi caractérisée, la trajectoire du développement de l'UJM traduit tout d'abord l'importance croissante, accordée par les équipes de direction successives, à la structuration de la politique scientifique de l'établissement ${ }^{19}$. Afin de soutenir cette dynamique, des priorités ont été clairement affichées dans l'affectation des ressources de I'université. L'analyse des contrats de I'UJM confirme, sur un autre plan, l'ouverture progressive de l'université (surtout à partir de 1995) sur son environnement externe à des

\footnotetext{
${ }^{19}$ Non sans lien avec l'intégration, par la tutelle, des ex-contrats quadriennaux de recherche à la
}

fins partenariales. En l'absence de l'énoncé d'objectifs en matière d'augmentation des flux étudiants, ce mouvement semble s'inscrire davantage dans la construction d'une identité visible que par la recherche d'un régime de croissance conjointe à proprement parler.

A aucun moment cependant, le contenu des cinq contrats d'établissement étudiés ne reflète explicitement les catégories traditionnelles de la stratégie (spécialisation différenciation sur des bases traditionnellement disciplinaires d'une part, poursuite d'un effet de masse critique par fusions ou alliances d'autre part). Aussi, les résultats de cette analyse thématique nous conduisent-ils à formuler la deuxième proposition suivante : la contractualisation n'a pas réussi à s'imposer comme levier efficace des stratégies universitaires, parce que les choix stratégiques des établissements ne sont pas clairement explicités dans leurs contrats. Pour autant, il ne s'agit pas pour nous d'affirmer ici que I'UJM n'a jamais poursuivi de stratégies depuis 1991. Le fait est qu'elles n'ont jamais été distinctement spécifiées dans la rédaction de ses contrats. Le contenu de ces derniers s'émancipe-t-il pour autant des attentes et orientations de l'Etat en matière de politique générale d'enseignement supérieur et de recherche ? Tel est le troisième volet que nous proposons d'aborder pour finir.

négociation des contrats d'établissement au milieu des années 1990. 


\subsection{La contractualisation : démarche stratégique réelle ou mise en scène stratégique ?}

Le tableau 3 ci-après met en parallèle les objectifs assignés par le ministère à l'UJM au moment de la préparation de trois de ses contrats d'une part, et les réponses apportées par l'université telles qu'elles apparaissent dans chacun des textes finaux d'autre part.

\section{Tableau 3 : les effets de l'isomorphisme institutionnel sur la stratégie de l'UJM}

\begin{tabular}{|c|c|c|}
\hline & $\begin{array}{l}\text { OBJECTIFS ASSIGNES PAR LE MINISTERE EN AMONT DE LA } \\
\text { PREPARATION DU PROJET }\end{array}$ & $\begin{array}{l}\text { OBJECTIFS FINALEMENT AFFICHES PAR L'UJM DANS LE } \\
\text { CONTENU DE SON CONTRAT }\end{array}$ \\
\hline $\begin{array}{l}\text { Contrat } \\
1999-2002\end{array}$ & $\begin{array}{l}\text { - Affirmer des priorités communes par une } \\
\text { politique de site entre les établissements et les } \\
\text { collectivités concernés } \\
\text { - Développer les coopérations inter-universitaires } \\
\text { sur site ou en réseaux thématiques } \\
\text { - Définir des axes stratégiques autour du } \\
\text { positionnement national et international de } \\
\text { l'établissement, de l'évolution de l'offre de } \\
\text { formation, de l'amélioration de la vie étudiante, } \\
\text { des choix en matière de politique scientifique } \\
\text { - En tirer les conséquences sur la politique de } \\
\text { gestion des ressources humaines, d'organisation et } \\
\text { de mobilisation des moyens }\end{array}$ & $\begin{array}{l}\text { - Contribuer à l'élargissement du pôle } \\
\text { scientifique régional grâce à une recherche lisible } \\
\text { et reconnue au niveau international } \\
\text { - Poursuivre l'adaptation aux exigences } \\
\text { socioéconomiques du bassin d'emploi } \\
\text { - Donner aux étudiants et aux personnels un } \\
\text { cadre de vie attractif et agréable qui renforce le } \\
\text { sentiment d'appartenance à une communauté } \\
\text { - Développer des partenariats avec les grands } \\
\text { organismes nationaux de recherche, les } \\
\text { universités et grandes écoles voisines } \\
\text { - Rassembler les activités et les ressources sur } 4 \\
\text { sites principaux }\end{array}$ \\
\hline $\begin{array}{l}\text { Contrat } \\
2003-2006\end{array}$ & $\begin{array}{l}\text { - Améliorer l'offre de formation et la réussite de } \\
\text { l'ensemble des publics accueillis } \\
\text { - Renforcer les collaborations inter-établissements } \\
\text { - Développer et moderniser la documentation } \\
\text { - Valoriser la politique de recherche en cohérence } \\
\text { avec l'offre de formation } \\
\text { - Conduire une gestion prévisionnelle des } \\
\text { ressources humaines } \\
\text { - Intégrer la promotion de l'égalité des chances } \\
\text { entre les hommes et les femmes } \\
\text { - Fournir les axes qui serviront d'appui aux } \\
\text { discussions contractuelles (formation, recherche, } \\
\text { international, GRH, patrimoine, TICE) }\end{array}$ & $\begin{array}{l}\text { - Développer de nouveaux partenariats en } \\
\text { cohérence avec les objectifs de renforcement de } \\
\text { ses domaines d'excellence, de progression de la } \\
\text { mobilité étudiante et enseignante, } \\
\text { d'amélioration de la qualité d'accueil des publics } \\
\text { étrangers } \\
\text { - Privilégier la politique scientifique des réseaux } \\
\text { et des équipes multi-sites } \\
\text { - Coopérer davantage avec les universités } \\
\text { régionales comme au niveau européen } \\
\text { - Renforcer la politique de suivi des emplois } \\
\text { - Développer des outils de pilotage des systèmes } \\
\text { d'information }\end{array}$ \\
\hline $\begin{array}{l}\text { Contrat } \\
2007-2010\end{array}$ & $\begin{array}{l}\text { - Vérifier la cohérence entre politique de recherche } \\
\text { et grandes orientations structurantes de l'offre de } \\
\text { formation dans une perspective de maîtrise de la } \\
\text { gestion prévisionnelle de l'emploi } \\
\text { - Examiner les complémentarités et les } \\
\text { partenariats entre les différents établissements de } \\
\text { la région } \\
\text { - Proposer une offre scientifique et pédagogique } \\
\text { de qualité répondant aux besoins de qualification } \\
\text { des étudiants s'inscrivant d'emblée dans une } \\
\text { vision internationale }\end{array}$ & $\begin{array}{l}\text { - Donner priorité à la politique scientifique } \\
\text { (renforcement des logiques de pôles } \\
\text { thématiquestransversaux) } \\
\text { - Développer les coopérations et réseaux } \\
\text { pédagogiques aux niveaux régional, national et } \\
\text { international } \\
\text { - Réorganiser l'offre de formations en privilégiant } \\
\text { une politique de co-habilitation avec les } \\
\text { établissements d'enseignement supérieur } \\
\text { régionaux } \\
\text { - Se positionner plus largement en réponse sur } \\
\text { les appels d'offres européens }\end{array}$ \\
\hline
\end{tabular}


La lecture de ce tableau laisse a priori peu de doutes quant à l'effet des orientations ministérielles sur le développement de l'UJM. De fait, le processus de contractualisation observé peut être envisagé comme une mise en scène (une conformation) stratégique visant pour l'essentiel à satisfaire aux exigences de la tutelle (consistant en particulier à mieux articuler la cohérence entre axes de recherche et offre de formation; à développer la mise en réseau des établissements en promouvant des partenariats multi-sites ; à maîtriser les ressources et moyens employés en professionnalisant les outils de pilotage). Si l'on ajoute à cela le fait que les objectifs énoncés dans les circulaires et autres lettres en provenance du ministère s'adressent le plus souvent indifféremment à l'ensemble des établissements d'une même vague, alors la politique contractuelle menée en France depuis une quinzaine d'années s'apparente davantage à un outil au service d'une stratégie nationale qu'à un instrument qui ouvre des perspectives de développement stratégique singulières pour les universités. D'où l'énoncé de notre troisième et dernière proposition : la contractualisation n'a pas réussi à s'imposer comme levier efficace des stratégies universitaires, parce que sa pratique relève d'un processus d'isomorphisme institutionnel qui vise principalement à répondre aux attentes de la tutelle.

\section{Conclusion}

La vision traditionnelle du fonctionnement des universités, empreint à la fois de collégialité dans les prises de décision, de jeux de pouvoir entre acteurs académiques autonomes et d'autorité symbolique du leadership universitaire, n'encourage a priori pas l'étude de la stratégie de telles institutions. Pourtant, depuis une vingtaine d'années marquées par un double contexte de globalisation et de massification des systèmes d'enseignement supérieur, on assiste tout autant à la transposition de méthodes issues du "monde des affaires" dans le management universitaire, qu'à la promotion d'un modèle d'organisation plus entrepreneurial pour les universités. S'agissant du cas français, cette tendance coïncide avec la mise en œuvre d'une politique contractuelle entre chaque université et son ministère de tutelle, dont un récent rapport d'information du Sénat indique qu'elle n'a pas vraiment joué le rôle attendu de levier des stratégies universitaires. Pour quelles raisons en arrive-t-on à ce constat après vingt années de contractualisation ?

Trois propositions sont apportées en réponse à cette problématique, fondées sur l'analyse approfondie de la trajectoire contractuelle d'une université française entre 1991 et 2010. Ainsi selon nous, la contractualisation n'a pas réussi à s'imposer comme levier efficace des stratégies universitaires : parce qu'elle a essentiellement été envisagée comme vecteur pour obtenir des ressources supplémentaires ; parce que les choix stratégiques des établissements ne sont pas clairement explicités dans leurs contrats; parce que sa pratique relève d'un processus $\mathrm{d}^{\prime}$ 'isomorphisme institutionnel qui vise principalement à répondre aux attentes de la tutelle.

Ces résultats sont néanmoins le fruit d'une analyse dont il convient de souligner les principales limites. La première d'entre-elles tient sans doute au caractère situé de nos travaux. De ce point de vue, le problème n'est pas tant de souligner l'impossible généralisation de nos conclusions, que d'évoquer la singularité de l'université retenue : de création récente, pluridisciplinaire, localisée à proximité de pôles universitaires majeurs (Lyon et Grenoble) et de taille moyenne, I'UJM trouve sans doute dans sa nature et son histoire les fondements même de sa trajectoire stratégique passée et à venir. C'est pourquoi, nous avons employé le terme de proposition pour souligner à la fois le caractère contextuel (mais aussi potentiellement systémique) des observations issues du cas de l'UJM. Par ailleurs, notre analyse des conditions d'élaboration des différents contrats repose exclusivement sur des entretiens réalisés avec une catégorie particulière d'acteurs universitaires : bien que les anciens présidents 
rencontrés aient joué un rôle majeur dans la vie de I'UJM entre 1991 et 2010, il nous faut néanmoins être conscient de la singularité de leur angle de vue.

Cela étant et pour élargir le périmètre de notre réflexion, quelles pourraient être les implications managériales de notre travail ? Nous en identifions principalement une, qui concerne la question de l'évolution des formes de leadership universitaire en France. Le renforcement progressif du pouvoir de décision stratégique entre les mains des présidents d'université et de leurs équipes n'est pas spécifique au cas stéphanois, mais concerne l'ensemble du système français depuis la loi sur les libertés et responsabilités des universités de 2007. Ce phénomène va changer d'échelle à compter de la mise en œuvre de la loi de 2013 relative à l'enseignement supérieur et à la recherche, qui prévoit qu'un seul contrat soit désormais signé au niveau de chaque communauté d'universités et établissements (CUE). Dès lors selon nous, les futurs contrats de site ne gagneront pas (ou plus) à être considérés comme la copie qu'un Président de CUE remettrait à son Ministre afin d'obtenir les bons points de sa tutelle. L'enjeu qui consistera à faire des futurs contrats de site, si ce n'est le dispositif de co-construction de I'action stratégique interuniversitaire, tout du moins le vecteur d'appropriation d'une vision stratégique par la communauté académique, nous paraît de ce point de vue bien plus important. Une telle évolution tiendra tout autant dans la capacité de la tutelle publique à relâcher la contrainte d'un cadre trop standardisé en amont de la préparation des contrats, que dans la volonté des équipes de direction des communautés d'universités et établissements de mettre en œuvre suffisamment tôt des processus réellement collectifs dans la préparation de leurs projets de développement. Dans le cas contraire, quel sens continuer à donner à un processus contractuel dont il n'y a visiblement, depuis le passage progressif des universités aux compétences élargies en 2009, plus grand chose à attendre en matière d'allocation de ressources humaines ou budgétaires pour les établissements?

\section{Bibliographie}

ASKLING B., KRISTENSEN B., 2000, "Towards the learning organisation: implications for institutional governance and leadership ", Higher Education Management and Policy, vol.12, n², p.17-44.

BARGH C., BOCOCK J., SCOTT P., SMITH D., 2000, University Leadership: The Role of the Chief Executive, Open University Press, Londres.

BARTH I., 2012, "MDDDO dans les organisations : I'Université en route pour l'Oscar ? ", Colloque de I'Institut Psychanalyse \& Management, ClermontFerrand

BARTOLI A., 2002, " Ouvrir la boîte noire de I'Université française : une condition nécessaire à son évolution ? ", in CLIQUET G., ORANGE G. (dir.), Organisations privées, organisations publiques : mélanges Robert Le Duff, Presses Universitaires de Rouen, p.53-78.

BONACCORSI A., DARAIO C., 2007, " Theoretical perspectives on university strategy ", in BONACCORSI A., DARAIO C. (eds), Universities and Strategic Knowledge Creation: Specialisation and Performance in Europe, Edward Elgar Publishing, Cheltenham, p. 3-30.

BUCKLAND R., 2009, "Private and Public Sector Models for Strategies in Universities ", British Journal of Management, vol.20, n4, p. 524-536.

CHEVAILLIER T., 2002, « University governance and finance: the impact of changes in resource allocation on decision making structures ", in AMARAL A., JONES G.A., KARSETH B. (eds), Governing Higher Education: National Perspectives on Institutional Governance, Kluwer Academic Publishers, Dordrecht, p. 87-98.

CLARK B.R., 1998, Creating Entrepreneurial Universities: Organizational Pathways of Transformation, Pergamon, Oxford.

COHEN M.D., MARCH J.G., 1974, Leadership and Ambiguity: The American College President, McGraw-Hill, New-York.

DAVIES J.L., 2001, « The emergence of entrepreneurial cultures in European universities ", Higher Education Management and Policy, vol.13, $\mathrm{n}^{\circ} 2$, p. 27-46. 


\section{Politique contractuelle et stratégies universitaires : le rendez-vous manqué ?/ Hervé GOY}

DELANTY G., 2001, " The university in the knowledge society ", Organization, vol.8, $\mathrm{n}^{\circ} 2$, p.149-153.

DIMAGGIO P.J., POWELL W.W., 1983, " The iron cage revisited: institutional isomorphism and collective rationality in organizational fields ", American Sociological Review, vol.48, n², p. 147160.

ETZKOWITZ H., WEBSTER A., GEBHARDT C., TERRA B., 2000, " The future of the university and the university of the future: evolution from ivory tower to entrepreneurial paradigm ", Research Policy, vol.29, n², p. 313-330.

GOY H., 2013, " Proposition d'un mode opératoire pour caractériser la nature entrepreneuriale des universités ", in LEVY-TADJINE T., SU Z. (coord.), Entrepreneuriat, PME durables et réseaux sociaux, ouvrage en I'honneur de Robert Paturel, L'Harmattan, Paris, p. 345-355.

JOHNSON G., WHITTINGTON R., SCHOLES K., FRERY

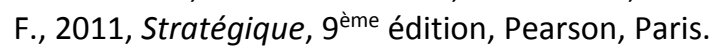

KIRBY D.A., GUERRERO M., URBANO D., 2011, «Making universities more entrepreneurial: development of a model ", Canadian Journal of Administrative Sciences, vol.28, n³, p. 302-316.

MAILHOT C., SCHAEFFER V., 2009, "Les universités sur le chemin du management stratégique ", Revue Française de Gestion, vol.35, $\mathrm{n}^{\circ} 191$, p. 33-48.

MALLET D., BALME P., RICHARD P., 2002, Réglementation et management des universités françaises, Berger-Levrault, Paris.

McNAY I., 1995, "From the Collegial Academy to Corporate Enterprise: the Changing Cultures of Universities ", in SCHULLER T. (ed), The Changing University?, Open University Press, Londres, p. 105115.

MEYER J., ROWAN B., 1977, " Institutionalized organizations: formal structure as myth and ceremony ", American Journal of Sociology, vol.83, $n^{\circ} 2$, p. 340-363.

MIGNOT-GERARD S., 2003, " Le leadership et le gouvernement dans l'analyse des organisations universitaires : deux notions à déconstruire ", Politiques et Gestion de l'Enseignement Supérieur, vol.15, n², p. 147-177.

MINTZBERG H., 1982, Structure et dynamique des organisations, Editions d'Organisation, Paris.

MUSSELIN C., 1997, « Les universités à l'épreuve du changement : préparation et mise en œuvre des contrats d'établissement ", Sociétés Contemporaines, vol.28, $\mathrm{n}^{\circ} 28$, p. 79-101.

MUSSELIN C., 2001, La longue marche des universités françaises, Presses Universitaires de France (collection Sciences Sociales et Sociétés), Paris.

NAJ R., HAMBRICK D.C., CHEN M-J., 2007, " What is strategic management, really? Inductive derivation of a consensus definition of the field ", Strategic Management Journal, vol.28, $n^{\circ} 9$, p. 935-955.

PFEFFER J., SALANCIK G.R., 1978, The External Control of Organizations: a Resource Dependence Perspective, Harper \& Raw, New-York.

TABATONI P., JARNIOU P., 1975, Les systèmes de gestion: politiques et structures, Presses Universitaires de France, Paris.

WEICK K.E., 1976, " Educational organizations as loosely coupled systems ", Administrative Science Quarterly, vol.21, n¹, p. 1-19. 
Annexe 1 : La trajectoire de l'UJM revisitée à l'aune de ses contrats d'établissement

1991-1994 : une 1ère contractualisation en contexte de croissance des effectifs étudiants

Pour faire face à la croissance des effectifs étudiants, le développement de l'offre de formations constitue l'axe central de la politique de l'UJM au début des années 1990. La carte des diplômes est sensiblement élargie : création de filières professionnalisantes, délocalisation d'enseignements de premier cycle sur le site roannais, mise en place de passerelles interdisciplinaires (en termes de réorientation des étudiants en situation d'échec à l'accès aux études médicales notamment). Au total, ce sont 15 nouveaux diplômes de second et troisième cycle qui sont créés (dont une filière complète pour la formation d'ingénieurs). De même, l'accent est mis sur l'amélioration de l'accueil et de l'orientation des étudiants (en particulier par l'organisation de journées portes ouvertes), ainsi que sur le besoin d'extension des surfaces bâties $\left(+8^{\circ} 000 \mathrm{~m}^{2}\right.$ d'infrastructures prévus sur la période du contrat). Le développement de I'UJM sur la période 1991-1994 s'accompagne, enfin, de la création de 90 emplois (enseignement et encadrement confondus).

1995-1998 : la nécessaire structuration du pilotage des activités au niveau de l'université

La croissance importante des effectifs étudiants (+50\% entre 1990 et 1995) engendre des phénomènes de seuils organisationnels, qui nécessitent de revisiter le système de pilotage de I'UJM. L'accent est mis sur le développement et la structuration des activités de formation : réorganisation de la filière technologique, développement des formations en alternance, renforcement des fonds documentaires. Les prémisses d'une politique scientifique d'établissement apparaissent : les activités de recherche sont catégorisées selon trois axes (sciences et techniques ; gestion et communication ; sport et santé). L'accent est mis sur l'identité de I'UJM (sa place et ses missions dans sa région d'implantation; son image et sa présence dans son agglomération). Plusieurs partenariats sont envisagés : avec d'autres universités de la région (pour la diffusion de nouvelles technologies éducatives ou le suivi de l'insertion professionnelle des étudiants), avec les milieux professionnels et institutionnels locaux (pour faciliter le rapprochement université - entreprises), avec d'autres établissements d'enseignement supérieur à l'étranger (pour favoriser la mobilité étudiante).

\section{9-2002 : l'affichage des ambitions scientifiques de l'établissement}

Trois défis sont identifiés dans ce troisième contrat : contribuer à l'élargissement du pôle scientifique régional grâce à une recherche lisible et reconnue au niveau international ; poursuivre la démocratisation de l'enseignement supérieur et l'adaptation aux exigences socio-économiques $\mathrm{du}$ bassin d'emploi ; donner aux étudiants et aux personnels un cadre de vie attractif et agréable qui renforce le sentiment d'appartenance à une communauté. La stagnation des inscriptions étudiantes est un facteur de contexte important et clairement identifié par I'UJM sur cette période. Par ailleurs, la part consacrée à la politique de recherche prend véritablement toute sa place dans ce troisième volet (renforcement de la politique de pôles scientifiques; développement des activités de valorisation de la recherche). Sur le plan de l'organisation spatiale, un plan de remembrement des ressources sur quatre sites géographiques principaux est défini. Enfin, la nécessité de maintenir et/ou développer des partenariats (avec les grands organismes nationaux de recherche, les 
universités et grandes écoles voisines) est réaffirmée.

\section{3-2006 : le besoin de collaborer pour exister}

Le principal fait marquant de ce quatrième contrat touche à la recherche affichée de partenariats. Dans un contexte de stabilisation de ses effectifs à partir de la rentrée 2000 (aux alentours de 13.000 étudiants), I'UJM cherche en effet à mettre en cohérence ses partenariats avec ses objectifs de renforcement de ses domaines d'excellence, de progression de la mobilité étudiante et enseignante, ainsi que d'amélioration de la qualité de l'accueil des publics étrangers. En matière de politique scientifique, compte tenu de la taille réduite de la majorité de ses laboratoires, I'UJM souhaite ainsi privilégier la politique des réseaux et des équipes multisites. Sur le plan de la formation, de nouvelles coopérations sont envisagées : avec les universités de la région pour développer la validation des acquis de l'expérience; au niveau européen pour les actions de politique intracommunautaire ou dirigées vers des pays tiers. De multiples objectifs de structuration des principaux secteurs d'activités sont également déclinés : renforcement de la politique de suivi des emplois ; développement d'outils de pilotage des systèmes d'information.

\section{7-2010 : la recherche de visibilité comme} enjeu majeur

A partir de 2005 et jusqu'à la mise en œuvre de son cinquième contrat, I'UJM se trouve confrontée au double défi de la taille et de la lisibilité : si le nombre d'étudiants inscrits à I'UJM diminue significativement (-6\%) entre les rentrées universitaires 2007 et 2008, l'évolution démographique ne suffit pas à expliquer ce phénomène, les universités voisines n'enregistrant pas la même tendance. Dans ce contexte, I'UJM détermine deux axes principaux : la politique scientifique comme priorité (renforcement des trois pôles optique et vision ; sciences, ingénierie et santé ; territoires et mutations), et une large ouverture sur les coopérations et réseaux régionaux, nationaux et internationaux (recherche de co-habilitation de formations). Ainsi, le constat de faiblesse des effectifs inscrits dans certaines disciplines conduit l'université à réorganiser son offre de formations, en privilégiant une politique de cohabilitation avec les établissements régionaux. De même, l'ouverture de la politique scientifique est envisagée sous l'angle d'une participation accrue aux appels d'offres européens, impliquant l'association de plusieurs partenaires scientifiques au niveau du continent. 